\title{
Realidade virtual no controle da dor em pacientes pediátricos queimados
}

\author{
Virtual reality in pain control in burnt pediatric patients
}

\section{Realidad virtual en el control del dolor en pacientes pediátricos quemados}

Leticia Maues Marques ${ }^{1 *}$, Marcos Vinícius da Conceição Furtado ${ }^{1}$, Augusto Cezar Ferraz da Costa1, Jhensela Silva dos Prazeres ${ }^{1}$, Amanda Gabrielle dos Santos Amaral ${ }^{1}$, Jaqueline Pinheiro da Silva ${ }^{2}$, Mycaeli Oliveira Alves ${ }^{3}$, Pedro Henrique de Sousa Miranda ${ }^{4}$, Jeicyanne Holanda de Vasconcelos ${ }^{4}$, Emille Amanda da Conceição Rodrigues ${ }^{5}$.

\section{RESUMO}

Objetivo: Revisar estudos que relatam o uso da realidade virtual e sua relação com o controle da dor em pacientes pediátricos queimados. Métodos: Trata-se de uma revisão integrativa de literatura. O levantamento foi feito no mês de outubro de 2020. As buscas foram realizadas nas bases de dados Biblioteca Virtual em Saúde, Phisiotherapy Evidence Database, Cochrane Library, Scientific Electronic Library Online e PubMed. Para rastreamento dos artigos indexados nas bases de dados foram utilizados os descritores burns, virtual reality, pain e children. Resultados: Foram incluídos na revisão sete estudos que utilizaram a realidade virtual como recurso não farmacológico no controle da dor de pacientes pediátricos queimados. Os estudos foram organizados em forma de quadro para apresentação dos principais achados, além dos objetivos e métodos de intervenção dos estudos. Considerações finais: A utilização da realidade virtual é um recurso indispensável no controle da dor em pacientes queimados, o qual precisa ser explorado, principalmente no público infantil, grupo em que a abordagem lúdica é bem aceita, resultando na redução da sensação dolorosa durante a realização de atividades e procedimentos terapêuticos necessários.

Palavras-chave: Queimaduras, Realidade virtual, Dor, Crianças.

\section{ABSTRACT}

Objective: Review studies that report the use of virtual reality and its relationship with pain control in pediatric burnt patients. Methods: It is an integrative literature review. The survey was carried out in October 2020. The searches were conducted in the Virtual Health Library, Phisiotherapy Evidence Database, Cochrane Library, Scientific Electronic Library Online and PubMed databases. For screening the articles indexed in the databases, the descriptors burns, virtual reality, pain and children were used. Results: Seven studies that used virtual reality as a non-pharmacological resource in pain control in pediatric burnt patients were included in the review. The studies were organized in the form of a table to present the main findings, in addition to the objectives and intervention methods of the studies. Final considerations: The use of virtual reality is an indispensable resource in pain control in burnt patients, which needs to be explored, especially in children, a group in which the playful approach is well accepted, resulting in the reduction of pain sensation during the performance of necessary therapeutic activities and procedures.

Keywords: Burns, Virtual reality, Pain, Children.

\section{RESUMEN}

Objetivo: Revisar estudios que reportan o utilizan la realidad virtual y su relación con el control del dolor en pacientes pediátricos quemados. Métodos: Es una revisión integradora de la literatura. La investigación se realizó en octubre de 2020. Mientras busca las bases de datos realizadas en la Biblioteca Virtual de Salud, la Base de Datos de Evidencia de Fisioterapia, la Biblioteca Cochrane, la Biblioteca Electrónica Científica en Línea y PubMed. Para el seguimiento de los artículos indexados en las bases de datos se utilizaron los descriptores quemaduras, realidad virtual, dolor y niños. Resultados: Se incluyen siete estudios en la revisión que utilizarán la realidad virtual como recurso no farmacológico para controlar el dolor de los pacientes pediátricos quemados. Los estudios se organizan en forma de tabla para la presentación de los puntos principales, junto con los objetivos y métodos de intervención de los estudios. Consideraciones finales: El uso de la realidad virtual es un recurso indispensable en el control del dolor em pacientes quemados, o que necesita ser explorado, especialmente en niños, un grupo en el que el acercamiento lúdico es bien aceptado y resulta en la reducción de sensaciones dolorosas durante la realización de Actividades y procedimientos terapéuticos necesarios.

Palabras clave: Quemaduras, Realidad virtual, Dolor, Niños.

\footnotetext{
${ }^{1}$ Escola Superior da Amazônia (ESAMAZ), Belém - PA. *E-mail: leticiamaues01@gmail.com

2 Universidade do Estado do Pará (UEPA), Belém - PA.

${ }^{3}$ Faculdade Paraense de Ensino (FAPEN), Belém - PA.

${ }^{4}$ Universidade Federal do Pará (UFPA), Belém - PA.

5 Faculdade UNINASSAU, Belém - PA.
} 


\section{INTRODUÇÃO}

As queimaduras são consideradas lesões ou traumas, acarretadas por condições externas, térmicas, químicas, elétricas ou radioativas, que podem provocar danos pequenos, grandes ou totais à pele e seus anexos, no entanto, quando acontece um trauma mais agressivo, pode atingir camadas mais profundas, lesionando, dessa forma, tecidos subcutâneos, sistema muscular, tendões e ossos (VIANA BS, et al., 2019). A priori, o trauma, dependendo do grau, pode até mesmo resultar em óbito (SALES MSC, et al., 2015).

Além do extenso período de internação, os danos provocados por queimaduras resultam em graves sequelas, o que requer um período prolongado de acompanhamento terapêutico pós alta hospitalar. Dessa forma, a queimadura pode ser apontada como uma agressão avassaladora, com importância ligada não somente à alta incidência, mas também, em especial, aos danos causados pela lesão que, por consequência, apresentam capacidade de desencadear sequelas funcionais, estéticas e psicológicas, além de elevada taxa de mortalidade (ARAGÃO JA, et al., 2012).

Os dados epidemiológicos disponíveis sobre a incidência de queimaduras durante um ano, são estimados em torno de 500 mil nos Estados Unidos da América (EUA), 10 a 15 mil internações hospitalares ao ano na Alemanha e 700 a 800 mil novos casos na Índia. Em relação aos dados epidemiológicos do Brasil, de acordo com os acidentes de queimaduras por ano, estima-se que seja em torno de um milhão de vítimas, sendo que apenas 100 mil pessoas procuram serviço médico para atendimento, porém cerca de 2.500 indivíduos chegam a óbito, por resultado de consequências agravantes diretas ou indiretas das queimaduras. Além do mais, de acordo com estudos do Sistema Único de Saúde (SUS) do Brasil, tais agravos são responsáveis por elevadas ocupações de internações hospitalares, cerca de 30 mil por ano (DIAS LDF, et al., 2015).

No Brasil, a gravidade das queimaduras é classificada pelos seguintes critérios: pequeno, médio e grande queimado. Pequeno queimado: $1^{\circ}$ e $2^{\circ}$ grau com até $10 \%$ da área do corpo atingida; Médio queimado: $1^{\circ}$ e $2^{\circ}$ grau afetando de $10 \%$ até $25 \%$ ou $3^{\circ}$ grau com até $10 \%$ da região corporal comprometida pela queimadura ou trauma na região da mão e pé; Grande queimado: $1^{\circ}$ e $2^{\circ}$ grau com região comprometida maior que $26 \%$ ou de $3^{\circ}$ grau maior que $10 \%$ no local danificado e, também, atingindo a região do períneo, a qual se encontra os órgãos genitais, além do mais, sabe-se que a região torácica é o local mais afetado em torno de $28,8 \%$ dos pacientes (SALES MSC, et al., 2015).

Além disso, as queimaduras estão entre as principais causas externas de óbito registradas, depois de outras causas violentas, correspondendo a um dos principais tipos de acidentes envolvendo crianças, ocupando a quarta causa de óbito, depois de acidentes de trânsito, afogamentos e quedas. Por outro lado, ainda ocupa também a sétima causa em admissão hospitalar (ARAGÃO JA, et al., 2012).

Os avanços no cuidado prestado ao paciente queimado estão diretamente ligados ao maior índice de sobrevida de indivíduos com queimaduras graves. Todavia, esses avanços estão aderidos também à maior ocorrência de sequelas que podem, de alguma forma, interferir na funcionalidade e, consequentemente, qualidade de vida desses pacientes. Logo, a atenção ao paciente queimado deve contemplar não apenas os aspectos físicos individuais, mas também questões psicológicas, emocionais e até sociais (ARAGÃO JA, et al., 2012).

É importante ressaltar que, os cuidados iniciais não abrangem imediatamente a ferida, voltando-se assim para cuidados que envolvam a manutenção da permeabilidade das vias aéreas do paciente, reposição de fluídos, além do controle da dor. Dessa forma, após reestabelecimento de vias aéreas, estabilidade hemodinâmica e redução da dor, ou ainda, medidas que visem a prevenção de agravamentos, inicia-se o processo do tratamento das feridas ocasionadas pela queimadura (ROSSI LA, et al., 2010).

O tratamento da ferida envolve a manutenção da perfusão tissular e, na medida do possível, preservação da integridade tecidual, mantendo-a higienizada e úmida. Além de que, o tratamento de uma ferida ocasionada por queimadura é norteado por medidas preventivas de infecções e traumas, procedimentos que influenciam positivamente o processo de cicatrização e assistência voltada à manutenção da mobilidade e funcionalidade da região afetada. Ademais, deve ser considerada a avaliação e atualização da imunização antitetânica (ROSSI LA, et al., 2010). 
Nesse sentido, a finalidade de todo o cuidado das feridas ocasionadas por queimaduras é a cicatrização em tempo favorável, visando o mínimo de complicações. Para isso, entre muitas intervenções de saúde, o ganho de tais resultados requer procedimentos de higienização propícia da ferida e a realização adequada de curativos. No entanto, há muitos fatores que influenciam no processo de cicatrização de feridas ocasionadas por queimaduras, entre os quais pode-se destacar a idade avançada, o estado nutricional, além de infecções e doenças como insuficiência vascular e diabetes mellitus (ROSSI LA et al., 2010).

A idade é considerada um agravante extremamente importante, o qual nos idosos está mais relacionado à maior dificuldade às adaptações fisiológicas, já nas crianças este agravante está mais relacionado com a exposição ao meio ambiente e o volume corporal (MARTINS VC, et al., 2020).

A sensação dolorosa no indivíduo com queimadura normalmente está relacionada à contínua perda da integridade tecidual e a procedimentos que estimulam de forma direta o leito da lesão, onde os nociceptores da epiderme e da derme foram destruídos (SCAPIN S, et al., 2017).

Paralelo a isso, se torna indispensável que a equipe multiprofissional analise as características da queimadura, para então decidir a melhor conduta terapêutica, objetivando o alívio do sofrimento do paciente durante os procedimentos indicados no tratamento. Tendo em vista a dimensão da queimadura, para o controle e alívio da dor são utilizados tratamentos farmacológicos e não farmacológicos (SCAPIN S, et al., 2017).

Diante disso, um grande recurso não farmacológico que está sendo muito utilizado e estudado atualmente é a realidade virtual (RV), a qual é classificada como analgesia distrativa, possibilitando a simulação de um cenário real, no qual, por meio de uma interface indivíduo-máquina o paciente corresponde ao que é simulado por meio da potencial capacidade de interação e imersão produzidas, de forma que a atenção seja desviada do ambiente real para o ambiente virtual com a exposição de animações tridimensionais, em tempo real, promovendo assim a interação e, consequentemente, distração durante o atendimento, sendo aplicada principalmente nas condutas que geram um estímulo maior à dor (CARICCHIO MBM, 2017; SCAPIN S, et al., 2017).

Ademais, já se tem evidências internacionais que confirmam os resultados favoráveis desse tratamento em pacientes queimados, voltados para a diminuição da percepção de intensidade dolorosa e ansiedade (FARIAS MB, et al., 2019). Sobretudo, evidências científicas têm sido enfoque da neurociência moderna para compreender as respostas cerebrais de crianças às situações por elas vivenciadas, com análise não só em experiências positivas, mas também negativas. Nesse âmbito, a RV é considerada um meio facilitador promissor de tratamento de várias afecções (CARICCHIO MBM, 2017).

Nesse sentido, o uso constante deste recurso tecnológico vem sendo muito indicado para a redução da intensidade dolorosa durante procedimentos terapêuticos em pacientes pediátricos vítimas de queimaduras, diminuindo o processamento da dor através da redução da atividade cerebral com enfoque na situação vivenciada, de modo que o recurso esteja associado às técnicas de distração e entretenimento no cuidado humanizado, possibilitando assim o desenvolvimento da criança não só no ambiente terapêutico, mas também no social (FARIAS MB, et al., 2019).

Além disso, a utilização da RV está relacionada a possíveis colaborações no desempenho de funções cognitivas básicas, de forma que a atenção, concentração, memória, planejamento e resolução de problemas sejam influenciados (FARIAS MB, et al., 2019). Logo, ao levar em consideração a importância da ludicidade para o público infantil, vários profissionais utilizam o brincar como estratégia fundamental, apta para melhor compreender e atender à criança (CARICCHIO MBM, 2017).

Todavia, é necessária a realização de mais pesquisas sobre a utilização dessa nova tecnologia, visto que seus efeitos se dão de forma benéfica. Sendo assim, o presente estudo tem como objetivo: revisar estudos que relatam o uso da realidade virtual e sua relação com o controle da dor em pacientes pediátricos queimados. 


\section{MÉTODOS}

Trata-se de uma revisão integrativa de literatura. O levantamento foi feito no mês de outubro de 2020 . As buscas foram realizadas nas bases de dados Biblioteca Virtual em Saúde (BVS), Phisiotherapy Evidence Database (PEDro), Cochrane Library, Scientific Electronic Library Online (Scielo) e PubMed.

Para rastreamento dos artigos indexados nas bases de dados supramencionadas foram utilizados descritores em Inglês cadastrados em Ciências da Saúde (DeCS): burns, virtual reality, pain e children. Além disso, os descritores foram pesquisados de forma associada, utilizando o operador boleano: AND.

Os critérios de inclusão definidos para esta revisão foram: estudos primários (ensaios clínicos, relato de casos), estudos com texto completo, estudos publicados em Inglês ou português, estudos publicados nos últimos 10 anos e estudos que utilizaram a realidade virtual como terapia de controle da dor em paciente pediátrico queimado.

Foram excluídos estudos que não obedeceram ao desenho de estudo adotado (artigos de revisão, metanálises, guia de prática clínica), estudos com texto incompleto, outros idiomas de publicação, estudos com data de publicação inferior aos últimos 10 anos, duplicação de publicação e estudos que não utilizaram como método de intervenção no controle da dor a realidade virtual em contexto clínico de pacientes pediátricos queimados.

A busca e seleção dos estudos foi adaptada de acordo com os critérios do modelo de fluxograma Prisma 2009 Flow Diagram (MOHER D, et al., 2015) (Figura 1).

Figura 1 - Fluxograma das buscas e seleção dos artigos conforme PRISMA.

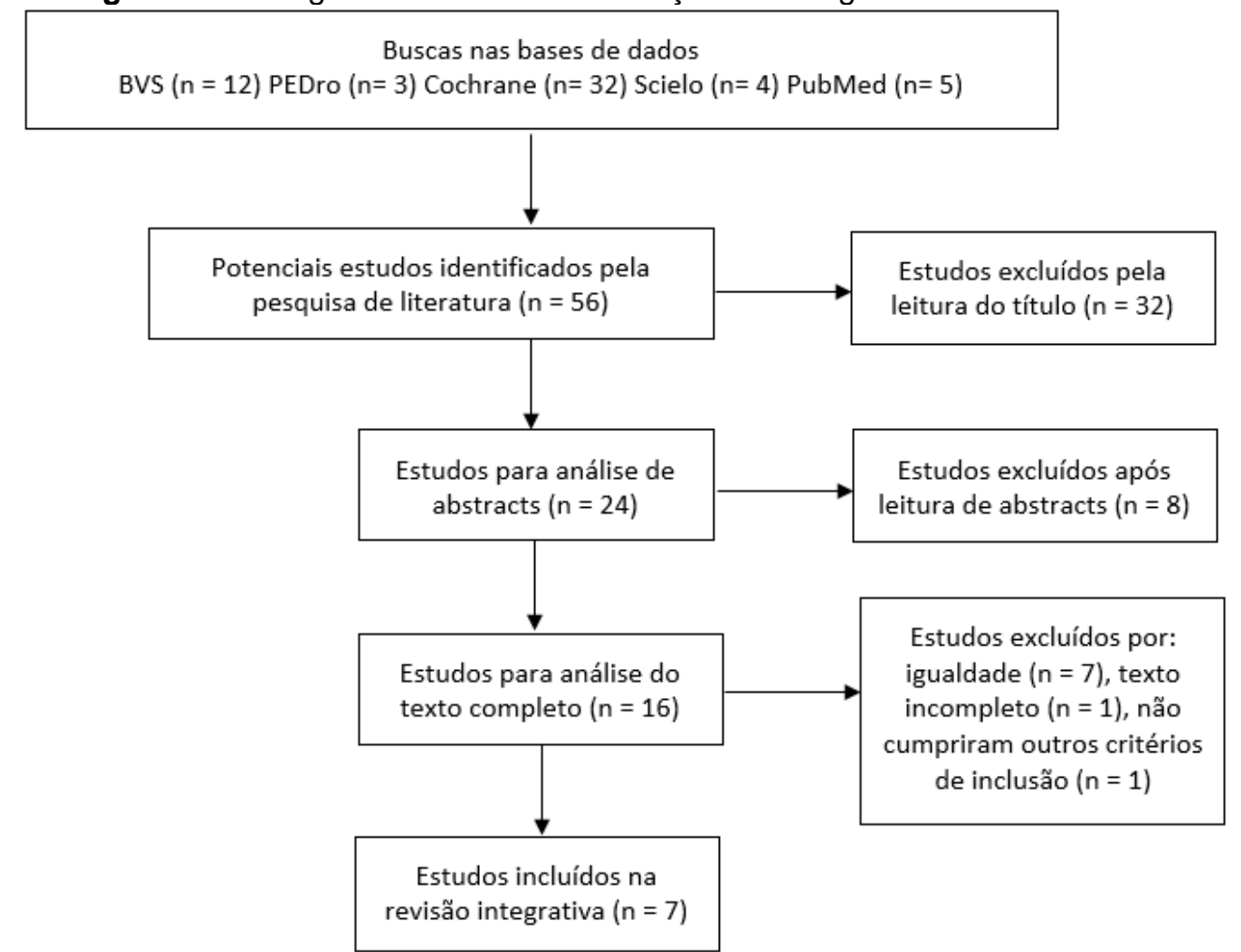

Fonte: Marques LM, et al., 2021.

\section{RESULTADOS}

Considerando os procedimentos metodológicos descritos anteriormente, os resultados da pesquisa foram sendo refinados ao longo do processo, em função dos critérios estabelecidos. Dessa forma, foram selecionados para a análise final 7 estudos, os quais foram estruturados por meio de resumo de suas principais características, bem como resultados com interesse para a temática em análise, apresentados no quadro seguinte (Quadro 1). 
Quadro 1 - Principais características e desfechos/resultados dos estudos selecionados.

\begin{tabular}{|c|c|c|c|c|c|}
\hline Autor/ano & Título & Objetivo & Amostra/método & Principais resultados das análises & Conclusão \\
\hline $\begin{array}{l}\text { BROWN N, } \\
\text { et al. (2013) }\end{array}$ & $\begin{array}{l}\text { Brincar e curar: ensaio } \\
\text { controlado randomizado } \\
\text { da eficácia da } \\
\text { intervenção DittoTM na } \\
\text { melhoria da } \\
\text { reepitelização em } \\
\text { queimaduras pediátricas }\end{array}$ & $\begin{array}{c}\text { Investigar o efeito de uma } \\
\text { intervenção não farmacológica } \\
\text { (DittoTM: um dispositivo médico } \\
\text { eletrônico portátil que fornece } \\
\text { preparação do procedimento e } \\
\text { distração) na reepitelização de } \\
\text { feridas por queimaduras agudas } \\
\text { pediátricas. }\end{array}$ & $\begin{array}{l}\text { Crianças de } 4 \text { a } 13 \text { anos. } \\
\text { GC: } 52 \text { participantes, submetidos } \\
\text { à preparação e distração padrão. } \\
\text { Gl: } 47 \text { participantes, submetidos à } \\
\text { preparação processual e a } \\
\text { distração multimodal. }\end{array}$ & $\begin{array}{l}\text { Os participantes do Gl tiveram o } \\
\text { tempo de reepitelização de } 2 \text { dias } \\
\text { mais rápidos em comparação ao GC, } \\
\text { além de diminuição no número de } \\
\text { trocas totais de curativos. Ademais, } \\
\text { após a segunda e terceira troca de } \\
\text { curativos o Gl apresentou níveis de } \\
\text { dores e ansiedade abaixo da média. }\end{array}$ & $\begin{array}{l}\text { O uso do dispositivo DittoTM possibilitou } \\
\text { aos pacientes um menor estresse durante } \\
\text { as mudanças de curativos, além dos } \\
\text { resultados positivos sobre o período de } \\
\text { reepitelização, se mostrando como uma } \\
\text { ferramenta útil para ser usada como } \\
\text { complemento de analgesia não } \\
\text { farmacológica para queimaduras } \\
\text { pediátricas. }\end{array}$ \\
\hline $\begin{array}{l}\text { KHADRA C, } \\
\text { et al. (2018) }\end{array}$ & $\begin{array}{l}\text { Ambiente de cúpula de } \\
\text { realidade virtual } \\
\text { baseado em projetor } \\
\text { para dor e ansiedade de } \\
\text { procedimentos em } \\
\text { crianças pequenas com } \\
\text { queimaduras: um } \\
\text { estudo piloto }\end{array}$ & $\begin{array}{l}\text { Avaliar a viabilidade e } \\
\text { aceitabilidade do processo de } \\
\text { estudo e o uso de RV para } \\
\text { gerenciamento de dor processual. }\end{array}$ & $\begin{array}{l}\text { Crianças de } 2 \text { meses a } 10 \text { anos } \\
\text { (15 participantes), as quais foram } \\
\text { submetidas a um ambiente de RV } \\
\text { através de uma cúpula que era } \\
\text { acionada no momento em que as } \\
\text { crianças eram sujeitas à } \\
\text { hidroterapia. }\end{array}$ & $\begin{array}{l}\text { Durante o processo os níveis álgicos } \\
\text { permaneceram abaixo da média com } \\
\text { o decorrer do desbridamento, visto } \\
\text { que este é um procedimento curto, } \\
\text { porém doloroso. Além disso, os níveis } \\
\text { de ansiedade também se mostraram } \\
\text { abaixo da média. }\end{array}$ & $\begin{array}{l}\text { Os resultados do ambiente de cúpula por } \\
\text { meio da realidade virtual se mostraram } \\
\text { benéficos como tratamento não } \\
\text { farmacológico no auxílio das intervenções } \\
\text { em questão. No entanto, estudos mais } \\
\text { elaborados serão necessários para } \\
\text { confirmar a real eficácia do ambiente de } \\
\text { cúpula de RV. }\end{array}$ \\
\hline $\begin{array}{l}\text { MILLER K, } \\
\text { et al. (2011) }\end{array}$ & $\begin{array}{l}\text { Uma nova abordagem } \\
\text { de tecnologia para o } \\
\text { tratamento da dor em } \\
\text { crianças com } \\
\text { queimaduras: um } \\
\text { estudo controlado } \\
\text { randomizado. }\end{array}$ & $\begin{array}{l}\text { Determinar se um protocolo de } \\
\text { distração multimodal de RV irá } \\
\text { reduzir a dor e sofrimento de } \\
\text { crianças de } 3 \text { a } 10 \text { anos } \\
\text { submetidas a procedimentos de } \\
\text { tratamento de queimaduras como } \\
\text { pacientes ambulatoriais, quando } \\
\text { comparadas com crianças } \\
\text { submetidas à Distração padrão. }\end{array}$ & $\begin{array}{c}\text { Crianças de } 3 \text { a } 10 \text { anos de idade } \\
\text { (40 participantes). } \\
\text { GC: procedimentos ambulatoriais } \\
\text { regulares de medicação e } \\
\text { distração. } \\
\text { Gl: distração multimodal, na qual } \\
\text { as crianças foram submetidas a } \\
\text { meios combinados de preparação } \\
\text { do procedimento e distração } \\
\text { padrão. }\end{array}$ & $\begin{array}{l}\text { O GI apresentou uma redução no } \\
\text { quadro de dor em comparação ao } \\
\text { GC, assim como no nível de } \\
\text { desconforto. Além disso, o grupo que } \\
\text { fez o uso de RV apresentou uma } \\
\text { melhora relevante de cicatrização das } \\
\text { feridas, que se deu em torno de } 15 \\
\text { dias, enquanto a do outro grupo se } \\
\text { deu em torno de } 18 \text { dias. }\end{array}$ & $\begin{array}{l}\text { Os resultados do estudo indicam o } \\
\text { significativo impacto positivo do uso } \\
\text { terapêutico de dispositivos de realidade } \\
\text { virtual, por meio de distração multimodal, } \\
\text { amenizando, dessa forma, os quadros } \\
\text { álgicos durante os procedimentos e } \\
\text { acelerando o processo de cicatrização dos } \\
\text { ferimentos. }\end{array}$ \\
\hline $\begin{array}{l}\text { SCHMITT Y, } \\
\text { et al. (2011) }\end{array}$ & $\begin{array}{l}\text { Um ensaio randomizado } \\
\text { e controlado de } \\
\text { analgesia de realidade } \\
\text { virtual imersiva durante } \\
\text { a fisioterapia para } \\
\text { queimaduras pediátricas }\end{array}$ & $\begin{array}{l}\text { Comparar a eficácia de analgesia } \\
\text { por RV imersiva adjuvante com a } \\
\text { do tratamento farmacológico } \\
\text { convencional, durante uma ou } \\
\text { mais sessões de fisioterapia pós- } \\
\text { queimadura no ambiente } \\
\text { hospitalar. }\end{array}$ & $\begin{array}{l}\text { Indivíduos de } 6 \text { a } 19 \text { anos (54 } \\
\text { participantes), submetidos à } \\
\text { fisioterapia pós-queimadura no } \\
\text { mínimo uma vez durante sua } \\
\text { estadia no hospital. Cada } \\
\text { participante recebeu em } \\
\text { determinado momento durante as } \\
\text { sessões somente analgesia } \\
\text { farmacológica padrão, já em outro } \\
\text { momento acrescentou-se a } \\
\text { utilização de RV. }\end{array}$ & $\begin{array}{c}\text { Os resultados do estudo } \\
\text { demonstraram que durante o uso da } \\
R V \text { os pacientes informaram } \\
\text { reduções significativas em relação à } \\
\text { experiência dolorosa no primeiro dia } \\
\text { de estudo }(\mathrm{n}=27-44 \%) \text {. Além disso, } \\
\text { a analgesia foi mantida com o uso } \\
\text { repetido de RV em várias sessões de } \\
\text { terapia. }\end{array}$ & $\begin{array}{c}\text { A realidade virtual é um agente } \\
\text { complementar não farmacológico de } \\
\text { grande relevância no auxílio de controle } \\
\text { da dor, ocasionada por lesões decorrentes } \\
\text { de queimaduras. }\end{array}$ \\
\hline
\end{tabular}




\begin{tabular}{|c|c|c|c|c|c|}
\hline Autor/ano & Título & Objetivo & Amostra/método & Principais resultados das análises & Conclusão \\
\hline $\begin{array}{l}\text { HOFFMAN } \\
\text { G, et al. } \\
\text { (2019) }\end{array}$ & $\begin{array}{c}\text { Realidade virtual } \\
\text { imersiva como um } \\
\text { analgésico adjuvante } \\
\text { não opióide para } \\
\text { crianças } \\
\text { predominantemente } \\
\text { latino-americanas com } \\
\text { grandes queimaduras } \\
\text { graves durante a } \\
\text { limpeza de feridas na } \\
\text { unidade de terapia } \\
\text { intensiva: um estudo } \\
\text { piloto }\end{array}$ & $\begin{array}{l}\text { Testar pela primeira vez, se a RV } \\
\text { imersiva pode servir como um } \\
\text { analgésico não opióide adjuvante } \\
\text { para crianças com grandes e } \\
\text { graves queimaduras durante a } \\
\text { limpeza de feridas na UTI, em um } \\
\text { centro regional de queimados nos } \\
\text { Estados Unidos, entre 2014-2016. }\end{array}$ & $\begin{array}{l}48 \text { participantes (6-17 anos). } \\
\text { Durante o tratamento de feridas, } \\
\text { em alguns períodos os } \\
\text { participantes jogaram SnowWorld, } \\
\text { um game 3D interativo em RV, em } \\
\text { outras partes do tratamento os } \\
\text { participantes não foram sujeitos à } \\
\mathrm{RV} \text {, com porções comparáveis ao } \\
\text { período de intervenção com RV } \\
\text { da mesma sessão de tratamento. }\end{array}$ & $\begin{array}{l}\text { Os participantes tiveram reduções } \\
\text { importantes na sensação } \\
\text { desagradável de dor com o uso de } \\
\text { RV durante os procedimentos de } \\
\text { limpeza de feridas. Ademais, os } \\
\text { participantes continuaram a relatar o } \\
\text { padrão previsto de dor mais baixa, } \\
\text { além de mais diversão com a RV, } \\
\text { durante várias sessões. }\end{array}$ & $\begin{array}{l}\text { A RV imersiva pode colaborar para a } \\
\text { redução da dor durante a limpeza de } \\
\text { feridas na UTI em pacientes pediátricos } \\
\text { considerados grandes queimados. No } \\
\text { entanto, sugere-se o desenvolvimento de } \\
\text { mais estudos sobre a temática. }\end{array}$ \\
\hline $\begin{array}{l}\text { KHADRA C, } \\
\text { et al. }(2020)\end{array}$ & $\begin{array}{l}\text { Efeitos de uma } \\
\text { realidade virtual híbrida } \\
\text { baseada em projetor na } \\
\text { dor em crianças } \\
\text { pequenas com } \\
\text { queimaduras durante } \\
\text { sessões de hidroterapia: } \\
\text { um sujeito interno } \\
\text { ensaio cruzado } \\
\text { randomizado }\end{array}$ & $\begin{array}{l}\text { Determinar se a distração de RV } \\
\text { híbrida combinada com o } \\
\text { tratamento farmacológico, fornece } \\
\text { maior redução da dor durante a } \\
\text { hidroterapia do que somente o } \\
\text { tratamento farmacológico padrão } \\
\text { em crianças de } 6 \text { meses a } 7 \text { anos } \\
\text { com queimaduras. }\end{array}$ & $\begin{array}{c}38 \text { participantes (6 meses a } 7 \\
\text { anos de idade). Os pacientes } \\
\text { foram sujeitos à hidroterapia } \\
\text { dividida em dois segmentos de } \\
\text { tratamento de feridas } \\
\text { proporcionais, com o uso de RV } \\
\text { híbrida durante um segmento e } \\
\text { sem RV híbrida durante o outro, } \\
\text { com ordem de tratamento } \\
\text { randomizada. }\end{array}$ & $\begin{array}{c}\text { A utilização da RV híbrida se } \\
\text { expressou de forma } \\
\text { significativamente relevante quanto à } \\
\text { diminuição dos níveis de dor, além de } \\
\text { aumentar o conforto dos pacientes } \\
\text { durante o procedimento. }\end{array}$ & $\begin{array}{l}\text { A RV híbrida se mostrou efetiva para a } \\
\text { redução da dor aguda em crianças } \\
\text { pequenas com queimaduras durante a } \\
\text { hidroterapia. Além disso, tais achados são } \\
\text { relevantes ao considerar que um } \\
\text { percentual elevado dos pacientes é } \\
\text { composto por crianças muito jovens. }\end{array}$ \\
\hline $\begin{array}{l}\text { SCAPIN S, } \\
\text { et al. (2017) }\end{array}$ & $\begin{array}{l}\text { Utilização da realidade } \\
\text { virtual no tratamento de } \\
\text { crianças queimadas: } \\
\text { relato de casos }\end{array}$ & $\begin{array}{l}\text { Relatar a eficácia do uso da RV } \\
\text { no alívio da dor na troca de } \\
\text { curativos de uma criança } \\
\text { queimada internada em Centro de } \\
\text { Tratamento ao Queimado (CTQ) } \\
\text { de referência no Sul do Brasil. }\end{array}$ & $\begin{array}{l}\text { Estudo de caso com } 1 \\
\text { participante (idade de } 9 \text { anos), a } \\
\text { criança foi submetida à RV } \\
\text { durante a troca de curativos, por } \\
\text { meio da utilização de óculos que } \\
\text { exibem imagem tridimensional e } \\
\text { som estereofônico. }\end{array}$ & $\begin{array}{l}\text { A utilização dos óculos de RV durante } \\
\text { a fase de troca de curativos foi } \\
\text { considerado de fácil aplicação, além } \\
\text { de boa aceitação pela criança, } \\
\text { resultando em redução dos níveis de } \\
\text { dor durante os procedimentos. }\end{array}$ & $\begin{array}{l}\text { As produções científicas direcionadas ao } \\
\text { uso da RV no Brasil ainda são escassas. } \\
\text { Diante disso, o seguinte estudo busca } \\
\text { estimular pesquisas com amostras } \\
\text { significativas em relação aos benefícios da } \\
\text { RV no controle da dor em pacientes } \\
\text { pediátricos queimados. }\end{array}$ \\
\hline
\end{tabular}

Legenda: GC: grupo controle; Gl: grupo intervenção; RV: realidade virtual; UTI: unidade de terapia intensiva; ECG: Escala de Classificação Gráfica.

Fonte: Marques LM, et al., 2021. 


\section{DISCUSSÃO}

As intervenções de saúde em pacientes pediátricos queimados requerem a inserção do lúdico, e entre os recursos atuais, a RV se destaca por ser um recurso extremamente atrativo e capaz de assegurar a atenção da criança, sendo fundamental para a distração e consequente redução álgica nos procedimentos padrões de reabilitação do paciente queimado (CARICCHIO MBM, 2017).

No estudo de Scapin SQ, et al. (2017), os autores relatam que, ao fazer o uso da RV durante a troca de curativos a criança percebia uma redução da intensidade álgica, em decorrência da sua atenção estar voltada para a atividade que deveria realizar. O estudo também observou que a criança se tornou mais colaborativa, houve melhora na aceitação dos procedimentos, dessensibilização álgica e aumentou a capacidade de enfrentar a situação, logo, o estudo constatou que tais fatores associados ao tratamento convencional medicamentoso são fundamentais para a melhora da qualidade de vida, ao reduzir o sofrimento do paciente.

Corroborando com essa percepção, Hoffman HG, et al. (2019) constatou que a imersão da criança na RV é capaz de reduzir a percepção da intensidade dolorosa durante os curativos realizados na Unidade de Terapia Intensiva. Neste estudo, a utilização de óculos para imersão durante o tratamento propiciou que os estímulos sensoriais e visuais fossem difundidos, ao invés da criança se concentrar no que se passava ao seu redor, o seu enfoque era voltado para o ambiente criado pela RV. Além disso, outro benefício a ser promovido pela RV é a "diversão" dos pacientes durante a intervenção, uma vez que o desenvolvimento de novos gráficos, a renderização, animação, o reconhecimento de voz e inteligência artificial garantem aumento da diversão e interação no tratamento, indicando indiretamente uma melhora no humor ou afeto com a experiência, facilitando o sucesso na reabilitação (FREITAS DM e SPADONI VS, 2019).

Ademais, o estudo de Khadra C, et al. (2020) identificou melhorias em relação a diminuição da dor e aumento do conforto durante a aplicação da RV nas sessões de hidroterapia em pacientes pediátricos vítimas de queimaduras. Visto que, o principal sintoma relatado pelos pacientes é a dor, pois os procedimentos terapêuticos incluem, de modo geral, o desbridamento, limpeza e mobilização, culminando na maximização álgica, sendo imprescindível que a equipe multiprofissional recorra às técnicas que visem a redução do desconforto. Nesse contexto, Silva A, et al. (2015) retrata que a associação de medicamentos analgésicos com a RV reduz a dor em suas três dimensões: sensorial, afetiva e cognitiva, um efeito que não é alcançado com a utilização somente dos fármacos.

Outrossim, é reconhecido que fatores psicológicos podem influenciar na potencialização da dor, Mathews $\mathrm{L}$ (2011) relata que a dor pode interferir na qualidade de vida da criança, afetando o humor, sono, apetite e participação social. Em pacientes com queimaduras a dor é muito associada à ansiedade que juntamente com outros fatores psicológicos influenciam no aumento da intensidade álgica, sendo assim, ao direcionar esses fatores na intervenção haverá diminuição da dor. Para certificar essa acepção, Schmitt Y, et al. (2011), ponderou a porcentagem de eficácia analgésica que a RV Imersiva promove, demonstrando ser clinicamente significativa na redução da intensidade de dor; com redução de $27 \%$ (dor sensorial), $44 \%$ (dor cognitiva) e $32 \%$ (dor afetiva), quando utilizada no tratamento de pacientes pediátricos internados com queimaduras.

Paralelo a isso, Brown NJ, et al. (2013) abordou em seu estudo os efeitos que o estresse, ansiedade e dor possuem no processo de cicatrização. Como resultado, a inclusão de RV na intervenção aos pacientes pediátricos com queimaduras promoveu o processo de reepitelização das feridas mais rápido, menor tempo nas trocas dos curativos e redução de 1 ponto nos escores de ansiedade e dor em relação ao grupo controle. Tais repercussões foram associadas à uma limpeza mais completa em decorrência da distração das crianças e a redução da dor e ansiedade também são incluídas como fatores que predispõem uma reepitelização mais acelerada.

Além disso, há uma necessidade cada vez mais frequente de métodos que controlem a dor sem causar tantos efeitos colaterais, pois o procedimento farmacológico padrão causa náuseas, vômitos, constipação, prurido, hiperalgesia induzida pelos opióides e interferência do sono (BROWN NJ, et al., 2013). Em contrapartida, tanto os achados de Scapin S, et al. (2017) quanto de Faber AW, et al. (2013) corroboram que efeitos colaterais como desorientação, cansaço visual e náuseas podem estar relacionados ao uso da RV, porém esses estudos ratificam que as probabilidades de efetivação desses sintomas são praticamente nulas 
e que não geram malefícios no tratamento, uma vez que essa prática demonstra pouco ou nenhum efeito colateral habitualmente.

A vista disso, Khadra C, et al. (2018) observou em sua pesquisa que apenas $20 \%$ das crianças que receberam a intervenção multimodal necessitaram de analgesia de resgate durante a hidroterapia, em $68,7 \%$ das vezes os profissionais de saúde relataram que auxiliou no controle da dor, em $55,6 \%$ a criança foi mais colaborativa e todos indicaram que usariam a RV novamente. Entretanto, essa pesquisa revelou que há discrepâncias entre os efeitos conforme a idade, pois crianças menores de 6 meses interagem melhor com brinquedos comuns do que com o ambiente de cúpula, assim como crianças maiores de 7 anos não conseguiram emergir na cuba de hidroterapia por não caberem.

Para mais, Rossi LA et al. (2010), alerta que a hidroterapia por imersão é útil em uma fase menos aguda, e em queimaduras não muito extensas, uma vez que a submersão promove a limpeza local e exsudato, para desbridamento de tecidos desvitalizados frouxos, permitindo a participação ativa do paciente no tratamento. Nesse sentido, Miller K, et al. (2011) obteve também redução nos escores de dor, além de reforçar evidências de que a tecnologia oferece inúmeros benefícios para o tratamento de queimaduras, visto que promove atividades multissensoriais inovadoras cognitivas e fisicamente envolventes ao paciente durante a execução dos procedimentos profissionais.

No estudo de Miller K, et al. (2011), também foi verificado que esse recurso é eficiente em desviar a atenção de imagens e sons que estimulam o medo e a dor durante os processos de trocas de curativos, reduzindo assim o impacto sensorial de resposta dolorosa e a ansiedade. Paralelo a isso, Parry l, et al. (2013) destaca que a RV Imersiva pode ser usada ainda para uma ampla gama de procedimentos médicos dolorosos, por apresentar um custo-benefício menor se comparada com alguns procedimentos farmacológicos, além disso, a praticidade de muitos sistemas de jogos também o torna uma ferramenta potencialmente eficiente para complementar um programa de terapia após a alta hospitalar.

Contudo, Brown NJ, et al. (2013) relata em seu estudo que, avaliar e intervir na intensidade da dor pediátrica é um desafio multiprofissional, uma vez que há, nesse público, algumas dificuldades de compreensão e expressão verbal dessa experiência. De tal modo, como terapia não farmacológica, a distração da RV é considerada mais eficaz que as distrações tradicionais passivas em decorrência da potencial imersão, interação e diversão geradas (JEFFS D, et al., 2014). Nesse contexto, este recurso é alvo de estudos relacionados ao alívio da dor em variadas situações, tendo como finalidade a mudança do modo como a dor é percebida pelo paciente, pois a dor caracteriza-se como um sentimento extremamente angustiante de elevada complexidade, não sendo integralmente compreendido (FREITAS DM e SPADONI VS, 2019).

Logo, referente aos artigos elencados neste estudo, em todos observou-se redução da dor, 2 estudos referiram diversão e redução da ansiedade, 2 estudos relataram diminuição da necessidade de medicamentos analgésicos, 1 abordou melhorias na cicatrização e 2 relatam diversão e distração durante o tratamento com RV. A explicação para os efeitos se deve às técnicas de distração que a RV proporciona, haja vista que são novos estímulos para serem processados pelo cérebro, reduzindo assim, a sua capacidade de processar os estímulos dolorosos, o que somado com a teoria de controle do portão da dor e as implicações que a atenção promove nesta, resulta em diminuição quanto à modulação, percepção e resposta à dor, que, consequentemente, reduz a sensação de ansiedade e melhora o processo cicatricial (JEFFS D, et al., 2014).

\section{CONSIDERAÇÕES FINAIS}

Ao considerar o que foi exposto anteriormente, pode-se supor que a RV é indispensável no controle da dor em pacientes pediátricos queimados, visto que os cuidados terapêuticos para estes pacientes são compostos por procedimentos extremamente dolorosos, todavia, essenciais para a recuperação clínica. Dessa forma, a RV caracterizada pela sua capacidade de imersão, distração e interação com o paciente é considerada um recurso analgésico não farmacológico promissor, o qual precisa ser explorado, principalmente no público infantil, grupo em que a abordagem lúdica é bem aceita. Contudo, ainda há poucos estudos sobre a temática, principalmente estudos brasileiros, logo, sugere-se a elaboração de mais pesquisas sobre a RV como alternativa não farmacológica complementar no controle da dor em pacientes pediátricos queimados. 


\section{REFERÊNCIAS}

1. ARAGÃO JA, et al. Estudo epidemiológico de crianças vítimas de queimaduras internadas na unidade de tratamento de queimados do hospital de urgência de Sergipe. Rev. Bras Cir Plást, 2012; 27(3): 379-82.

2. BROWN NJ, et al. Play and heal: Randomized controlled trial of DittoTM intervention efficacy on improving reepithelialization in pediatric burns Burns. Burns: Journal of the International Society for Burn Injuries, 2013; 40(2): 204-213.

3. CARICCHIO MBM. Tratar brincando: o lúdico como recurso da fisioterapia pediátrica no Brasil. Revista Eletrônica Atualiza Saúde. Rev. Eletrôn. Atualiza Saúde, 2017; 6(6): 43-57.

4. DIAS LDF, et al. Unidade de tratamento de queimaduras da Universidade Federal de São Paulo: estudo epidemiológico. Rev. Bras Cir Plást, 2015; 30(1): 86-95.

5. FABER AW, et al. Repeated use of immersive virtual reality therapy to control pain during wound dressing changes in pediatric and adult burn patients. J Burn Care Res, 2013; 34(5): 563-8.

6. FARIAS MB. A experiência da criança hospitalizada com a realidade virtual em procedimentos dolorosos. AL, Dissertação (Mestrado em Enfermagem) - Universidade Federal de Alagoas, Maceió, 2019; 87p.

7. FREITAS DM, SPADONI VS. A realidade virtual é útil para manejo da dor em pacientes submetidos a procedimentos médicos? Einstein (São Paulo), 2019; 17(2): 1-3.

8. HOFFMAN HG. et al. Immersive virtual reality as an adjunctive non-opioid analgesic for predominantly Latin American children with large severe burn wounds during burn wound cleaning in the Intensive Care Unit: A pilot study. Front Hum Neurosci, 2019; 8(13): 262.

9. JEFFS D, et al. Effect of virtual reality on adolescent pain during burn wound care. J of Burn Care Res, 2014; 35(5): 395-408.

10. KHADRA C, et al. Effects of a projector-based hybrid virtual reality on pain in young children with burn injuries during hydrotherapy sessions: A within-subject randomized crossover trial. Burns, 2020; 46(7): 1571-1584.

11. KHADRA C, et al. Projector-based virtual reality dome environment for procedural pain and anxiety in young children with burn injuries: a pilot study. Journal of Pain Research, 2018; 11: 343-353.

12. MATHEWS L. Pain in Children: Neglected, Unaddressed and Mismanaged. Indian Journal of Palliative Care, 2011; 17(Suppl): 70-73.

13. MARTINS VC, et al. Estudo epidemiológico dos pacientes vítimas de queimaduras, tratados em um ambulatório do hospital municipal na cidade de Anápolis. Rev. de Ciênc Méd Biol, 2020; 19(2): 282-286.

14. MILLER K, et al. A novel technology approach to pain management in children with burns: a prospective randomized controlled trial. Burns, 2011; 37(3): 395-405.

15. MOHER D, et al. Itens de relatório preferidos para revisões sistemáticas e meta-análises: a declaração PRISMA. Rev. Epidemiol. Serv. Saúde, 2015; 24(2): 335-342.

16. PARRY I, et al. Keeping up with video game technology: Objective analysis of Xbox Kinect (TM) and PlayStation 3 Move (TM) for use in burn rehabilitation. Burns: Journal of the International Society for Burn Injuries, 2013; 40(5): 1-8.

17. ROSSI LA, et al. Cuidados locais com as feridas das queimaduras. Rev Bras Queimaduras. 2010; 9(2): 54-9.

18. CARNEIRO MS, NUNES RD. Abordagem fisioterapêutica em queimados: um estudo de revisão no âmbito da terapia intensiva. Rev. Amazônia Science \& Health, 2015; 3(2): 30-35.

19. SILVA A, et al. A terapia da realidade virtual e a pessoa queimada: redução da dor nos cuidados à ferida - Uma revisão integrativa da literatura. Rev. Brasileira de Queimaduras, 2015; 14(1): 35-42.

20. SCAPIN S. Realidade virtual no tratamento da dor em criança queimada: Relato de caso. Rev Bras de Queimaduras, $2017 ; 16(1): 45-8$.

21. SCHMITT Y, et al. A Randomized, Controlled Trial of Immersive Virtual Reality Analgesia during Physical Therapy for Pediatric Burn Injuries. Burns, 2011; 37(1): 61-68.

22. VIANA BS, et al. Complicações respiratórias secundárias a lesões inalatórias em indivíduos queimados e atuação fisioterapêutica: uma revisão literária. Rev. Bras, Queimaduras, 2018; 17(1): 56-6. 